\title{
Rate Adaptation Based on Exposure Assessment Using Rectenna Output for WLAN Station Powered with Microwave Power Transmission
}

\author{
Shota YAMASHITA ${ }^{\dagger a)}$, Koichi SAKAGUCHI ${ }^{\dagger}$, Yong HUANG ${ }^{\dagger \dagger}$, Student Members, \\ Koji YAMAMOTO ${ }^{\dagger}$, Senior Member, Takayuki NISHIO ${ }^{\dagger}$, Member, \\ Masahiro MORIKURA ${ }^{\dagger}$, Fellow, and Naoki SHINOHARA ${ }^{\dagger \dagger}$, Member
}

\begin{abstract}
SUMMARY This paper proposes a rate adaptation scheme (RAS) for a wireless local area network (WLAN) station powered with microwave power transmission (MPT). A WLAN station attempting to transmit data frames when exposed to microwave radiation for MPT, experiences a reduction in the physical (PHY) layer data rate because frames are lost even when the carrier sense mechanism is used. The key idea of the proposed scheme is to utilize the output of the rectenna used for receiving microwave power. Using rectenna output, a WLAN station based on the proposed scheme assesses whether the station is exposed to microwave radiation for MPT. Then, using historical data corresponding to the assessment result, the station selects an appropriate PHY data rate. The historical data are obtained from previous transmission results, e.g., historical data pertaining to the data frame loss ratio. The proposed scheme was implemented and verified through an experiment. Experimental results showed that the proposed scheme prevents the reduction in the PHY data rate, which is caused by the use of historical data stored in a single memory. Thus, the proposed scheme leads to an improvement in the WLAN throughput.

key words: wireless local area network, rate adaptation, microwave power transmission, rectenna
\end{abstract}

\section{Introduction}

Microwave power transmission (MPT) [1] is a wireless power transmission technology in which electric power is transmitted via microwaves. MPT enables us to transmit microwave power over long distances, as compared with other wireless power transmission methods, such as electromagnetic induction, magnetic resonance, and other components. Realizing the batteryless operation of a device by using wireless power transmission methods, allows the elimination of power cables and a reduction in battery replacement costs. These advantages increase as the number of batteryless devices increases, especially in a wireless sensor network.

When microwave power is transmitted to a wireless station, the influence of MPT on data transmission has to be considered. In this work, we use an IEEE 802.11 wireless local area network (WLAN), which employs the carrier

Manuscript received January 9, 2015.

Manuscript revised April 17, 2015.

${ }^{\dagger}$ The authors are with the Graduate School of Informatics, Kyoto University, Kyoto-shi, 606-8501 Japan.

${ }^{\dagger}$ The authors are with the Research Institute for Sustainable Humanosphere, Kyoto University, Uji-shi, 611-0011 Japan.

a)E-mail: info14@imc.cce.i.kyoto-u.ac.jp

DOI: $10.1587 /$ transcom.E98.B.1785 sense mechanism. WLAN stations with a high received interference power level defer the transmission of data frames and are unable to receive any frames.

In [2], the authors conducted experiments in which microwaves with a frequency of $2.4 \mathrm{GHz}$ were transmitted to an IEEE 802.11g-based WLAN station in co-channel or adjacent channel operations. The results were as follows. Firstly, when the received interference power at the station is high, MPT interferes with the data transmission even when using an adjacent channel. Accordingly, the station is deterred from transmitting data frames due to the carrier sense mechanism. Secondly, exposure of the station to microwave radiation for MPT leads to the selection of a lower physical (PHY) layer data rate, the use of which is maintained even after exposure to the microwave radiation is discontinued. The reduction in the PHY data rate occurs because the station attempts to continue data transmission during exposure to the microwave radiation. However, the station does not receive acknowledge (ACK) frames because of the high interference power. Hence, a low PHY data rate is selected with a rate adaptation scheme (RAS) that is implemented on the station. Note that this problem occurs even when the station employs the carrier sense mechanism.

In most RASs, by using data related to previous transmission results, e.g., historical data of the data frame loss ratio, the PHY data rate for data transmission is selected. The purpose of using the historical data is to estimate current link quality, which depends on the distance between a data transmitter and a data receiver and on interference power at the data receiver. To match the link quality, the PHY data rate is selected, i.e., such that data frames transmitted at the selected PHY data rate are successfully received by the data receiver. Related to the estimation of the link quality, many previous studies have attempted to propose RASs, e.g., ARF [3], RBAR [4], Onoe [5], and Sample Rate [6]. In addition, some conventional RASs employ loss differentiation mechanisms that diagnose the cause of data frame loss as collision or link quality degradation, e.g., LD-ARF [7], CARA [8], LDRA [9], and ERA [10]. However, these conventional schemes are designed without the assumption that high interference power at the station causes PHY data rate reduction.

Some previous studies have proposed RASs capable of 
assessing whether a WLAN station is exposed to microwave radiation from devices using Bluetooth, ZigBee, or from microwave ovens. SGRA [11] and ARES [12] attempt to assess whether a WLAN station is exposed to microwave radiation based on both the signal-to-noise power ratio (SNR) and the data frame loss ratio. However, because of the exposure assessment based on the data frame loss ratio, when the data receiver experiences strong SNR degradation, the station erroneously detects that it is exposed to microwave radiation for MPT even when this is not the case. The cause of this is that the data frame loss ratio increases, not only because of exposure of the station to microwave radiation for MPT, but also because of SNR degradation at the data receiver. The increase in the data frame loss ratio with SNR degradation is experimentally demonstrated in [13].

In this paper, we propose an RAS based on exposure assessment using rectenna output for a WLAN station exposed to microwave radiation for MPT. Then, we carry out experiments to evaluate the performance of the system on which the proposed scheme is implemented. The rectenna was installed in the device powered with MPT, and converts the microwave power it receives into direct current power. The rectenna output enables a station based on the proposed scheme to assess whether it is exposed to microwave radiation for MPT. The historical data corresponding to the assessment result are used by the station to select an appropriate PHY data rate, where two independent memories, each of which contains a different set of historical data for rate adaptation, are prepared in advance.

The proposed scheme was implemented on a WLAN station by modifying a mac80211 device driver [14] that is widely used as a WLAN driver in Linux systems. This device driver uses two conventional RASs by default, i.e., PID [15] and Minstrel [16]. By modifying either PID or Minstrel, we implemented PID-based or Minstrel-based proposed schemes on a WLAN station.

This paper is organized as follows. In Sect. 2, we explain conventional RASs (PID and Minstrel) used in the mac80211 device drivers. Section 3 contains our proposal for an RAS with rectenna output. The experiments conducted to evaluate the performance are described in Sect. 4. In Sect.5, we discuss the advantages of the proposed scheme. Finally, we conclude this paper in Sect. 6.

\section{Conventional Rate Adaptation Schemes of mac80211 Device Driver}

This section presents a description of the two conventional default RASs of the mac80211 device driver, i.e., PID and Minstrel. In Sects. 2.1 and 2.2, we explain the operation of the station based on each of these RASs. Note that the performances of these algorithms have been investigated in [17] and [18].

\section{$2.1 \quad$ PID}

A station based on PID selects an appropriate PHY data rate based on the general proportional integral derivative controller such that the data frame loss ratio converges to a predetermined target value, $R_{\mathrm{L} \text {,target }}$. The station selects the PHY data rate every sampling period $T_{\text {PID }}$

The $k$ th rate selection operates as follows. First, the station calculates the data frame loss ratio between the $(k-1)$ th and $k$ th rate selections, $r_{\mathrm{L}}[k]$. Second, the station calculates the $k$ th error between $R_{\mathrm{L} \text {,target }}$ and $r_{\mathrm{L}}[k]$ (denoted by $e[k]$ ) as

$$
e[k]=R_{\mathrm{L}, \text { target }}-r_{\mathrm{L}}[k] .
$$

Third, the station calculates the difference between $e[k]$ and $e[k-1]$ (denoted by $\Delta e[k])$ as

$$
\Delta e[k]=e[k]-e[k-1] .
$$

Moreover, the station computes an exponential moving average of $e[k]$ (denoted by $\hat{e}[k]$ ) by using $e[k]$ and $\hat{e}[k-1]$ computed at the $(k-1)$ th rate selection as

$$
\hat{e}[k]=\frac{\left(\alpha_{\text {smooth }}-1\right) \hat{e}[k-1]+e[k]}{\alpha_{\text {smooth }}},
$$

where $\alpha_{\text {smooth }}$ is a smoothing factor $\left(\alpha_{\text {smooth }}>1\right)$. Fourth, the station computes an adjustment value, $v_{\text {adj }}[k]$, as

$$
v_{\text {adj }}[k]=C_{\mathrm{P}} e[k]+C_{\mathrm{I}} \hat{e}[k]+C_{\mathrm{D}} \Delta e[k]\left(1+\alpha_{\text {sharp }}\right),
$$

where $\alpha_{\text {sharp }}$ is a sharpening factor (if $\alpha_{\text {sharp }} \neq 0$, a fast response is achieved), and $C_{\mathrm{P}}, C_{\mathrm{I}}$, and $C_{\mathrm{D}}$ are proportional, integral, and derivative coefficients, respectively. Finally, if $v_{\text {adj }}[k] \leq-1$, the station decreases the PHY data rate. If $v_{\text {adj }}[k] \geq 1$, the station increases the PHY data rate, and if $-1<v_{\text {adj }}[k]<1$, the station maintains the current PHY data rate.

\subsection{Minstrel}

A station based on Minstrel selects an appropriate PHY data rate by using the previous data frame transmission performance such that the highest throughput performance is achieved. The station updates a rate table every sampling period $T_{\text {Minstrel }}$. The rate table indicates the PHY data rate based on the retransmission count.

The station updates the $k$ th rate table as follows. First, $\mathcal{N}$ is a set of rate indexes that indicate the supported rates, and $i$ is an element of $\mathcal{N}$, i.e., $i \in \mathcal{N}$. Let the PHY data rate corresponding to $i$ be denoted by $R(i)$. Second, the station calculates $p_{\mathrm{s}}(i)[k]$ for every $i$, which represents the estimated success probability of data frame transmission at $R(i)$ as follows. Note that the term "success" represents the situation in which the station transmits a data frame and then receives an ACK frame. Let $\Delta n_{\text {transmit }}(i)[k]$ denote the increased number of data frames transmitted at $R(i)$ between the $(k-1)$ th and $k$ th updates. Similarly, let $\Delta n_{\text {success }}(i)[k]$ denote the increased number of data frames transmitted successfully at $R(i)$ between the $(k-1)$ th and $k$ th updates. Using $\Delta n_{\text {transmit }}(i)[k]$ and $\Delta n_{\text {success }}(i)[k], p_{\mathrm{s}}(i)[k]$ is calculated as

$$
p_{\mathrm{s}}(i)[k]=\left(1-\alpha_{\text {scale }}\right) \frac{\Delta n_{\text {success }}(i)[k]}{\Delta n_{\text {transmit }}(i)[k]}
$$


Table 1 Minstrel rate table created at the $k$ th update.

\begin{tabular}{cccc}
\hline \multirow{2}{*}{$\begin{array}{c}\text { Retransmission } \\
\text { count }\end{array}$} & \multirow{2}{*}{ Normal rate } & \multicolumn{2}{c}{ Lookaround rate (generating $\left.i_{\text {rand }}[k] \in \mathcal{N}\right)$} \\
\cline { 3 - 4 } & $R\left(i_{\text {rand }}[k]\right) \leq R\left(i_{\text {best_tp }}[k]\right)$ & $R\left(i_{\text {rand }}[k]\right)>R\left(i_{\text {best_tp }}[k]\right)$ \\
\hline 0 & $R\left(i_{\text {next_best_tp }}[k]\right)$ & $R\left(i_{\text {best_tp }}[k]\right)$ & $R\left(i_{\text {rand }}[k]\right)$ \\
\hline 1 & $R\left(i_{\text {best_prob }}[k]\right)$ & $R\left(i_{\text {rand }}[k]\right)$ & $R\left(i_{\text {best_tp }}[k]\right)$ \\
\hline 2 & $R\left(i_{\text {baserate }}[k]\right)$ & $R\left(i_{\text {best_prob }}[k]\right)$ & $R\left(i_{\text {best_prob }}[k]\right)$ \\
\hline 3 & & $R\left(i_{\text {baserate }}[k]\right)$ & $R\left(i_{\text {baserate }}[k]\right)$ \\
\hline
\end{tabular}

$$
+\alpha_{\text {scale }} p_{\mathrm{s}}(i)[k-1]
$$

where $\alpha_{\text {scale }}$ is a scaling value $\left(0<\alpha_{\text {scale }}<1\right)$. Third, the station computes the throughput at $R(i)$ (denoted by $t p(i)[k]$ ) using the estimated maximum number of data frames transmitted successfully at $R(i)$ per unit time. Note that the symbol " $t p$ " represents "throughput." Fourth, the station determines the following three rate indexes.

- $i_{\text {best_tp }}[k]$ : the rate index of the PHY data rate that will achieve the highest throughput performance

- $i_{\text {next_best_tp }}[k]:$ the rate index of the PHY data rate that will achieve the second highest throughput performance

- $i_{\text {best_prob }}[k]$ : the rate index of the PHY data rate that will achieve the highest success probability of data frame transmission

These rate indexes are defined as

$$
\begin{aligned}
i_{\text {best_tp }}[k] & =\underset{i \in \mathcal{N}}{\arg \max } t p(i)[k], \\
i_{\text {next_best_tp }}[k] & =\underset{i \in \mathcal{N}, i \neq i_{\text {best_tp }}[k]}{\arg \max } t p(i)[k], \\
i_{\text {best_prob }}[k] & =\underset{i \in \mathcal{N}}{\arg \max } p_{\mathrm{s}}(i)[k],
\end{aligned}
$$

respectively. Finally, the station updates the rate table in Table 1, where $i_{\text {baserate }}$ represents the rate index of the lowest supported PHY data rate.

Using Table 1, the station selects an appropriate PHY data rate as follows. The "Normal rate" is used to transmit $90 \%$ of data frames and the remaining $10 \%$ are transmitted at the "Lookaround rate," for which the station generates a random rate index, $i_{\text {rand }}[k] \in \mathcal{N}$. The station then determines the PHY data rate for the current retransmission count as shown in Table 1.

\section{Rate Adaptation Scheme Based on Exposure Assess- ment Using Rectenna Output}

In this section, we propose an RAS based on exposure assessment using rectenna output. The design of this proposed scheme focuses on the use of historical data in both of the conventional RASs, i.e., PID and Minstrel. Recall that historical data are obtained from previous transmissions, e.g., historical data of the data frame loss ratio. This proposed scheme has the following two features: (i) the station assesses whether it is exposed to microwave radiation for MPT by using rectenna output power; (ii) the station selects an appropriate PHY data rate using historical data corresponding

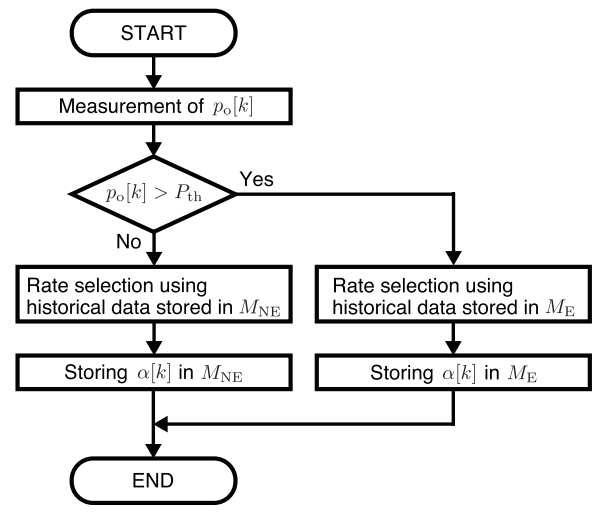

Fig. 1 Flowchart of $k$ th rate adaptation in the proposed scheme, where $\alpha[k]$ is the historical data obtained during $\left[t_{k-1}, t_{k}\right]$.

to the assessment result. The reason for using rectenna output power is twofold. First, a rectenna has been installed in a station powered with MPT and thus there is no need to install other devices to perform the exposure assessment. Second, the use of rectenna output power enables a station equipped with the rectenna to directly assess whether it is exposed to microwave radiation for MPT.

Figure 1 illustrates the flowchart of the proposed scheme. We explain $k$ th rate adaptation at the time $t_{k}$ as follows. Immediately before rate adaptation, the station measures the rectenna output power, $p_{\mathrm{o}}[k]$. Then, by using $p_{\mathrm{o}}[k]$, the station assesses whether it is exposed to microwave radiation for MPT. Let the power threshold be denoted by $P_{\text {th }} . P_{\text {th }}$ should be determined from the power the station receives from microwaves for MPT. The reasons are as follows. The output power of the rectenna that receives microwaves for communication is less than $1 \mu \mathrm{W}$. The reason is that the rectenna output power is not more than the maximum transmission power that limited to $10 \mathrm{~mW} / \mathrm{MHz}$ in Japan, and that at $2.4 \mathrm{GHz}$, the free space propagation loss at $1 \mathrm{~m}$ distance is equal to $40 \mathrm{~dB}$ in theory. On the other hand, the output power of the rectenna that receives microwaves for MPT is more than that of the rectenna that receives microwaves for communication, where, in this paper, we assume that the supplied power to the station is of the order of milliwatt.

Then, by using the historical data in the memory corresponding to the assessment result, the station selects an appropriate PHY data rate. The two independent memories in which the historical data for rate adaptation purposes are stored are denoted by $M_{\mathrm{E}}$ and $M_{\mathrm{NE}}$, where the subscripts "E" and "NE" represent "exposure" and "non-exposure," respectively. When $p_{\mathrm{o}}[k]>P_{\text {th }}$, the station determines that 
it is exposed to microwave radiation for MPT, after which it selects an appropriate PHY data rate using the historical data stored in $M_{\mathrm{E}}$, which contains the corresponding historical exposure data. At the same time, the historical data obtained during $\left[t_{k-1}, t_{k}\right]$ is stored in $M_{\mathrm{E}}$. On the other hand, when $p_{\mathrm{o}}[k] \leq P_{\mathrm{th}}$, the station determines that it is not exposed to microwave radiation for MPT and then selects an appropriate PHY data rate using the historical data stored in $M_{\mathrm{NE}}$, which contains the historical data corresponding to non-exposure to microwave radiation for MPT. At the same time, historical data obtained during $\left[t_{k-1}, t_{k}\right]$ are stored in $M_{\mathrm{NE}}$.

The scheme proposed in this paper does not define a concrete RAS; instead, it uses historical data corresponding to the state of exposure to microwave radiation for MPT. Hence, a station based on the proposed scheme is able to use RASs corresponding to the exposure assessment result. In the experiments described in Sect. 4, we implemented the proposed scheme on a station such that the PHY data rate is selected on the basis of a single conventional RAS regardless of the magnitude of $p_{\mathrm{o}}[k]$. This has the purpose of enabling a comparison with the conventional RAS. For example, in the "PID-based proposed scheme," $p_{\mathrm{o}}[k]$ is measured immediately before the $k$ th rate selection is measured, following which the PHY data rate is selected much in the same way as described in Sect. 2.1. Unlike in conventional PID, in the PID-based proposed scheme, historical data corresponding to the magnitude of $p_{\mathrm{o}}[k]$ is used to select the PHY data rate. When $p_{\mathrm{o}}[k]>P_{\text {th }}$, the PHY data rate is selected by using both $\hat{e}\left[k_{\text {last,E}}\right]$ and $e\left[k_{\text {last,E }}\right]$ that satisfy

$$
k_{\text {last }, \mathrm{E}}=\max _{k^{\prime} \in \mathcal{N}_{\mathrm{E}}} k^{\prime},
$$

where $\mathcal{N}_{\mathrm{E}}=\left\{n \mid p_{\mathrm{o}}[n]>P_{\mathrm{th}}, n=1, \ldots, k-1\right\}$. When $p_{\mathrm{o}}[k] \leq P_{\text {th }}$, the PHY data rate is selected by using both $\hat{e}\left[k_{\text {last }, \mathrm{NE}}\right]$ and $e\left[k_{\text {last }, \mathrm{NE}}\right]$ that satisfy

$$
k_{\text {last }, \mathrm{NE}}=\max _{k^{\prime} \in \mathcal{N}_{\mathrm{NE}}} k^{\prime},
$$

where $\mathcal{N}_{\mathrm{NE}}=\left\{n \mid p_{\mathrm{o}}[n] \leq P_{\mathrm{th}}, n=1, \ldots, k-1\right\}$.

\section{Experiments}

This section presents an explanation of the experiments in this paper. All measurements were performed in a radio frequency (RF) anechoic chamber to ensure the absence of any sources of wireless transmissions except for the system described in Sect. 4.1.

\subsection{Experimental Setup}

Figure 2 illustrates the experimental setup for the performance evaluation of the proposed scheme. The experimental system consists of the following three devices, an energy source (ES), a data transmitter (DT), and a data receiver (DR).

The details of each of these devices are as follows. The
ES intermittently transmits continuous microwaves for MPT to the DT and consists of a transmission antenna, an amplifier, and an RF signal generator. The microwaves generated by the RF signal generator are amplified via the amplifier, and are then transmitted from the transmission antenna to the DT.

The DT continuously transmits data frames to the DR. It consists of a commercial WLAN adapter (Logitec LANW150NU2AB, rt2800usb device driver), a Linux machine (Raspberry Pi Model B+ [19], Linux kernel 3.12.23+), a laptop (Apple MacBook Air, OS X 10.9.5), a microcontroller board (Arduino Uno board [20]), and a rectenna. The microcontroller board measures the rectenna output power and assesses whether the DT is exposed to microwave radiation for MPT, and then shares the information of the exposure assessment with the Linux machine. The Linux machine, which is remotely operated by the laptop, generates the data that are transmitted from the WLAN adapter.

The DR manages the network, receives the data frames transmitted from the DT, and captures the frames transmitted by the network as a packet sniffer. The DR consists of an access point (AP, Allied Telesis AT-TQ2403) and a laptop (Apple MacBook Pro, OS X 10.8.4). The AP generates and manages an IEEE 802.11g-based WLAN. The laptop receives the data frames transmitted from the DT via the AP and captures the frames transmitted from both the DT and the DR using its internal WLAN device. Note that the frame capture at the DR laptop is for the purpose of evaluating the throughput performance of the proposed scheme and is independent of the rate selection at the DT.

In this paper, the experiments were conducted only in the environment consisting of one DT and one DR, whereas the performance evaluation of the proposed scheme in an environment consisting of multiple DTs and DRs is beyond the scope of this paper. The reason is that the performance in the environment consisting of multiple nodes depends not only on the RAS but also on other factors, e.g., contention between the multiple nodes and the method of transmitting microwave power to the multiple nodes. In addition, we can verify the performancfe of the proposed scheme either in the environment consisting of multiple DTs and DRs or in the environment consisting of one DT and one DR. There are two reasons for this. One is that, in RASs, the PHY data rate for each pair of DT and DR is selected independently. The other one is that the two features of the proposed scheme, i.e., the exposure assessment using rectenna output and the use of historical data corresponding to the assessment result, are also performed independently on each DT.

\subsection{Parameters}

Figure 2 shows the position of each of the devices, and Fig. 3 shows the entire DT and the power transmission antenna of the ES. Figures 2 and 3 also show that the DT is placed in front of the transmission antenna, whereas the DR is placed behind the transmission antenna such that the microwave power transmitted from the ES only affects the DT. The DR 


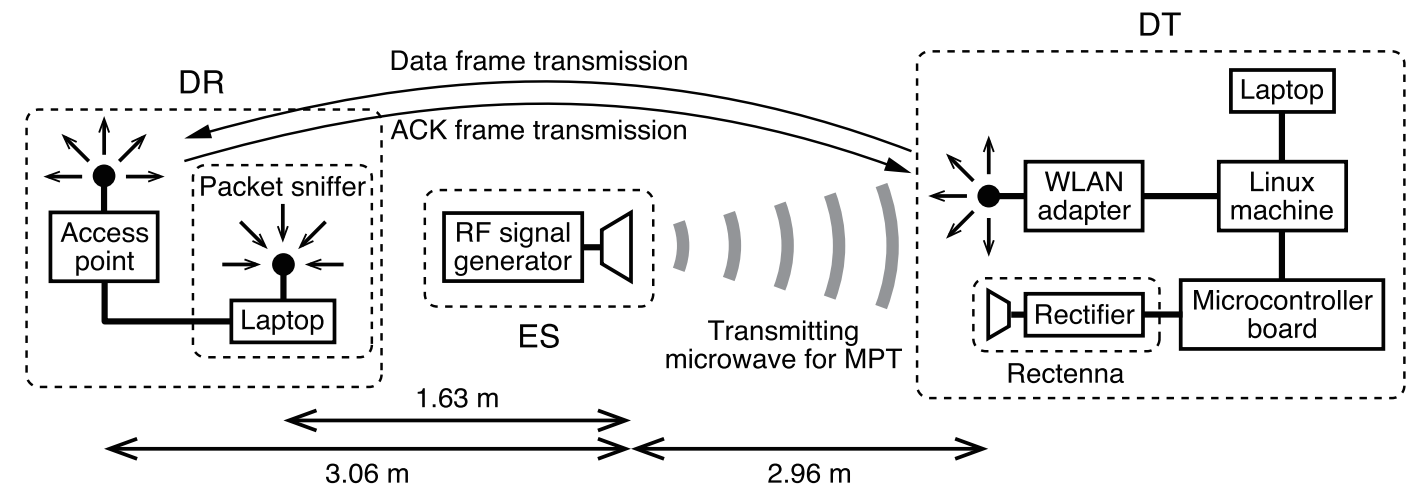

Fig. 2 Experimental setup.

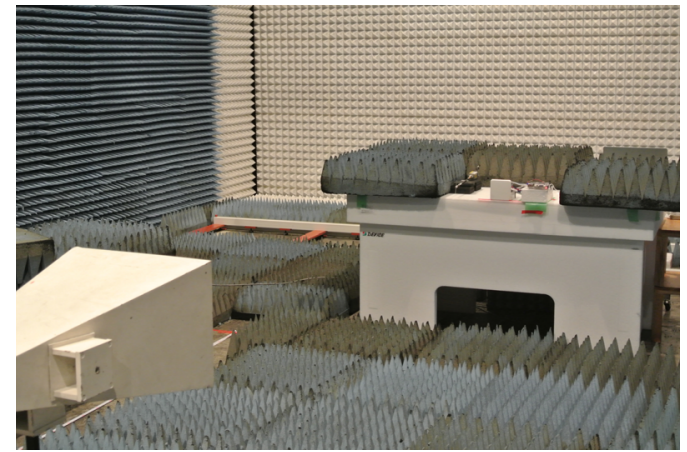

Fig. 3 DT and power transmission antenna.

AP, transmission antenna, and DT are placed in a straight line. The distances from the transmission antenna to the DR AP, DR laptop, and DT are $3.06 \mathrm{~m}, 1.63 \mathrm{~m}$, and $2.96 \mathrm{~m}$, respectively.

The ES repeatedly transmits continuous microwaves for MPT to the DT for $1.4 \mathrm{~s}$ and then pauses for $5.0 \mathrm{~s}$. Note that the duration of the microwave transmission, $1.4 \mathrm{~s}$, satisfies the following two conditions. It ensures that the DT does not become disassociated from the DR AP because of the consecutive non-reception of beacon frames, and, additionally, it ensures that the PHY data rate is reduced during this period. The central frequency of the microwaves is set to be $2.457 \mathrm{GHz}$, and the bandwidth is less than $2 \mathrm{kHz}$. The gain of the power transmission antenna is $16.4 \mathrm{dBi}$. When the ES transmits the microwaves, the transmission power of the ES is set to be $17.9 \mathrm{~W}$. In this case, the power density at each antenna position has the value listed in Table 2. Moreover, the DT rectenna output power when the ES transmited microwaves for MPT is $31 \mathrm{~mW}$, whereas the DT rectenna output power when the ES did not transmit microwaves for MPT is $0 \mathrm{~mW}$.

The DT transmits data frames at eight different PHY data rates: $6,9,12,18,24,36,48$, and $54 \mathrm{Mbit} / \mathrm{s}$. The central frequency for data transmission is set to be $2.457 \mathrm{GHz}$. Recall that MPT interferes with data transmission even when an adjacent channel is used for the purpose. The DT uses Iperf 2.0.5 [21] and generates user datagram protocol (UDP) data traffic with an offered load of $54 \mathrm{Mbit} / \mathrm{s}$, i.e., the
Table 2 Power density at each antenna when the ES transmits microwaves for MPT.

\begin{tabular}{ll}
\hline Antenna position & Power density \\
\hline DT rectenna & $0.86 \mathrm{~mW} / \mathrm{cm}^{2}$ \\
DT WLAN adapter & $0.92 \mathrm{~mW} / \mathrm{cm}^{2}$ \\
DR laptop & $1.33 \mu \mathrm{W} / \mathrm{cm}^{2}$ \\
DR AP & $0.97 \mu \mathrm{W} / \mathrm{cm}^{2}$ \\
\hline
\end{tabular}

network is saturated by traffic. The UDP datagram size is set to be $1,470 \mathrm{~B}$ and this size has no effect on the advantage of the proposed scheme because the throughput degradation discussed in this paper is caused by the ACK frame loss discussed in Sect. 1.

The proposed scheme was implemented on the DT Linux machine by modifying the mac 80211 device driver. Now, $P_{\text {th }}$ was set to be $6 \mathrm{~mW}$ taking both that the DT rectenna output power of $0 \mathrm{~mW}$ when the ES did not transmit microwaves for MPT and that the DT rectenna output power of $31 \mathrm{~mW}$ when the ES transmited microwaves for MPT into account. We emphasize that this value is for assessing that the rectenna output power is either $0 \mathrm{~mW}$ or $31 \mathrm{~mW}$ and does not sensitively affect the performance of the proposed scheme. In addition, to compare the role of the exposure assessment by using the rectenna output in the proposed scheme, we investigated the RAS without rectenna output for comparison as described in Sect. 4.3. As described in Sect. 3, regardless of the use of the rectenna output, the proposed scheme is implemented such that the PHY data rate is selected on the basis of either PID or Minstrel. The proposed scheme was modified as follows when implemented. As shown in Fig. 4, when the station determined that it was not exposed to microwave radiation for MPT in the $(k-1)$ th rate adaptation, but instead determines that it is exposed to microwave radiation for MPT in $k$ th rate adaptation, the station copies over the contents of $M_{\mathrm{NE}}$ to $M_{\mathrm{E}}$ immediately before the rate selection using $M_{\mathrm{E}}$. Even when we add these modifications to the proposed scheme, we are able to confirm its effectiveness because the content of $M_{\mathrm{NE}}$ shows the same behavior regardless of these modifications.

Based on the above discussion, we investigated the performance of each of the three PID-based and each of the three Minstrel-based RASs, i.e., the PID, PID-based pro- 


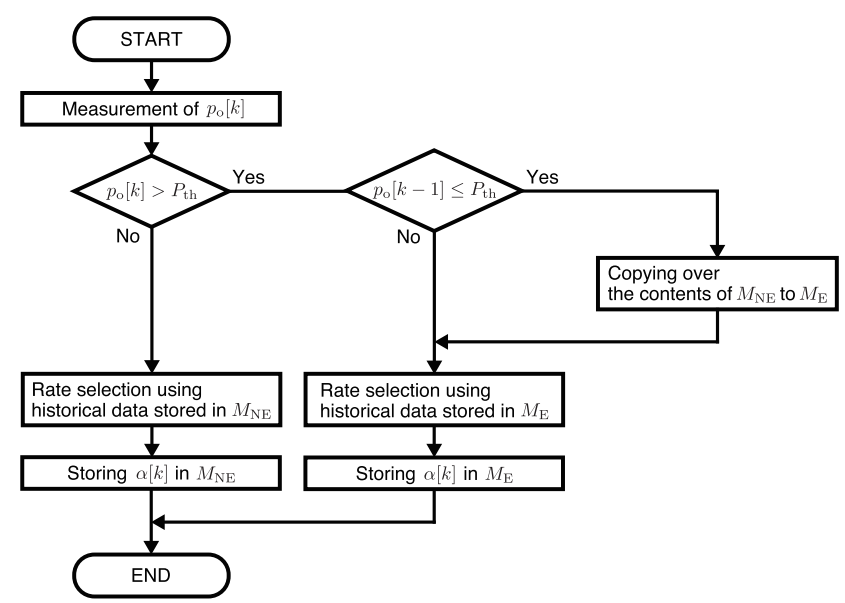

Fig. 4 Flowchart of $k$ th rate adaptation in the proposed scheme implemented for the experiments, where $\alpha[k]$ is the historical data obtained during $\left[t_{k-1}, t_{k}\right]$.

Table 3 PID default parameters.

\begin{tabular}{ccccccc}
\hline$T_{\mathrm{PID}}$ & $R_{\mathrm{L}, \text { target }}$ & $\alpha_{\text {smooth }}$ & $\alpha_{\text {sharp }}$ & $C_{\mathrm{P}}$ & $C_{\mathrm{I}}$ & $C_{\mathrm{D}}$ \\
\hline $125 \mathrm{~ms}$ & $14 \%$ & 8 & 0 & 15 & 9 & 15 \\
\hline
\end{tabular}

Table 4 Minstrel default parameters.

\begin{tabular}{cc}
\hline$T_{\text {Minstrel }}$ & $\alpha_{\text {scale }}$ \\
\hline $100 \mathrm{~ms}$ & 0.25 \\
\hline
\end{tabular}

posed scheme with and PID-based scheme without rectenna output for comparison, Minstrel, and Minstrel-based proposed scheme with and Minstrel-based scheme without rectenna output for comparison. The parameters used in both the PID-based and Minstrel-based RASs were initialized with the values in Tables 3 and 4, respectively, and were constant.

The experiments enabled us to evaluate the UDP throughput, PHY data rate used in the data frame transmission, and data frame loss ratio for each RAS. Firstly, the UDP throughput was estimated based on the number of ACK frames the DR laptop captured with Wireshark [22]. Recall that the frame capture at the DR laptop is for the purpose of evaluating the throughput performance of the proposed scheme and is independent of the rate selection at the DT. Even though the use of Iperf would enable us to calculate the UDP throughput, we estimated the UDP throughput with the captured frames. The reason is that the period of the UDP throughput estimation with the captured frames can be set shorter than that of the UDP throughput calculation with Iperf. Secondly, the PHY data rate in the PID-based RASs was measured on the DT Linux machine by using the debug function. On the other hand, the PHY data rate in the Minstrel-based RASs was estimated on the DR laptop by using captured data frames. In the Minstrel-based RASs, the reason for using captured data frames is that the PHY data rate used in each data transmission is unknown even when the debug function is used [16]. Lastly, the data frame loss ratio was measured on the DT Linux machine by using the debug function. We emphasize that the DT Linux machine was not able to completely obtain the data frame loss ratio because it did not have the required performance.

\subsection{Rate Adaptation Scheme Based on Exposure Assess-} ment Not Using Rectenna Output for Comparison

The performance of the proposed scheme was evaluated depending on whether the station uses rectenna output. For this reason we now explain the operation of an RAS without rectenna output for comparison. The station based on the scheme without rectenna output uses the results of previous data frame transmissions to assess whether the station is exposed to microwave radiation for MPT.

We explain the operation in the $k$ th rate adaptation at the time $t_{k}$ using the value of $r_{\mathrm{L}}[k]$ described in Sect.2.1. Recall that $r_{\mathrm{L}}[k]$ denotes the data frame loss ratio during $\left[t_{k-1}, t_{k}\right]$. Note that, even in the Minstrel-based scheme without rectenna output for comparison, $r_{\mathrm{L}}[k]$ is calculated. Let the number of data frames transmitted during $\left[t_{k-1}, t_{k}\right]$ be denoted by $\Delta n_{\text {transmit }}[k]$. In the same way as the proposed scheme, two memories $M_{\mathrm{E}}$ and $M_{\mathrm{NE}}$ are prepared. Using these memories, the $k$ th rate adaptation is performed as follows. When $r_{\mathrm{L}}[k-1]<50 \%$, if $r_{\mathrm{L}}[k] \geq 50 \%$, the station determines that it is exposed to microwave radiation for MPT, and then selects an appropriate PHY data rate using the historical data stored in $M_{\mathrm{E}}$ and then stores in $M_{\mathrm{E}}$ the historical data obtained during $\left[t_{k-1}, t_{k}\right]$. Then, the station continues to use historical data stored in $M_{\mathrm{E}}$ until $\Delta n_{\text {transmit }}[m] \geq 3 \Delta n_{\text {transmit }}[m-1]$ in the $m$ th rate adaptation for any $m>k$. On the other hand, when the station uses the historical data stored in $M_{\mathrm{E}}$ in the $(k-1)$ th rate adaptation, if $\Delta n_{\text {transmit }}[k] \geq 3 \Delta n_{\text {transmit }}[k-1]$, it determines that it is not exposed to microwave radiation for MPT, and then selects an appropriate PHY data rate using historical data stored in $M_{\mathrm{NE}}$ and stores in $M_{\mathrm{NE}}$ the historical data obtained during $\left[t_{k-1}, t_{k}\right]$. Then, the station continues to use the historical data stored in $M_{\mathrm{NE}}$ until both $r_{\mathrm{L}}[m-1]<50 \%$ and $r_{\mathrm{L}}[m] \geq 50 \%$ in the $m$ th rate adaptation for any $m>k$.

\subsection{Experimental Results}

Figure 5 shows both the UDP throughput and PHY data rate for each of the PID-based RASs. In addition, Fig. 6 shows both the data frame loss ratio and PHY data rate for each of the PID-based RASs. The red lines in Fig. 5 confirm that the PHY data rate is reduced when the DT is exposed to microwave radiation for MPT. Comparing both the UDP throughput and the PHY data rate in Fig. 5(a) with those in both Figs. 5(b) and 5(c), it is clear that there has been an improvement. Note that, in Figs. 5(b) and 5(c), there is a period during which the DT uses the lowest PHY data rate immediately after the MPT is discontinued. This is because the rate selection interval in the PID scheme is set to be $125 \mathrm{~ms}$.

Figure 7 shows both the UDP throughput and PHY data rate for each of the Minstrel-based RASs. In addition, Fig. 8 shows both the data frame loss ratio and PHY data rate for 


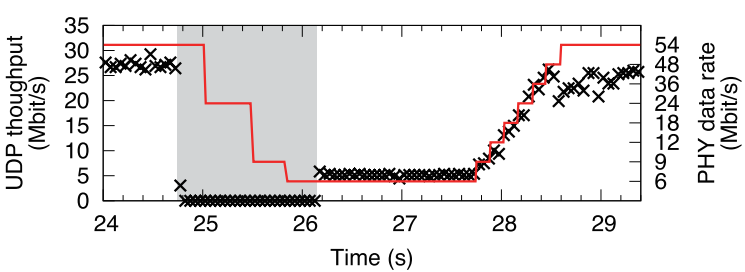

UDP throughput for every $0.050 \mathrm{~s} \quad \mathrm{X}$

(a) PID

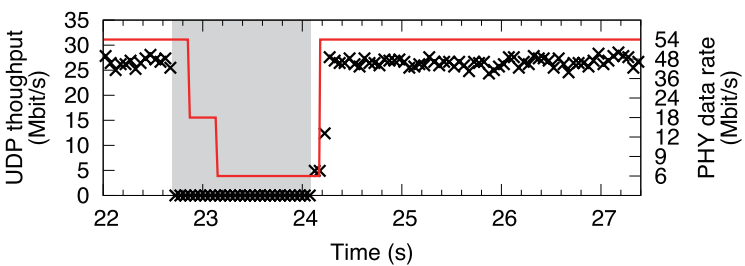

UDP throughput for every $0.050 \mathrm{~s} \times$

PHY data rate

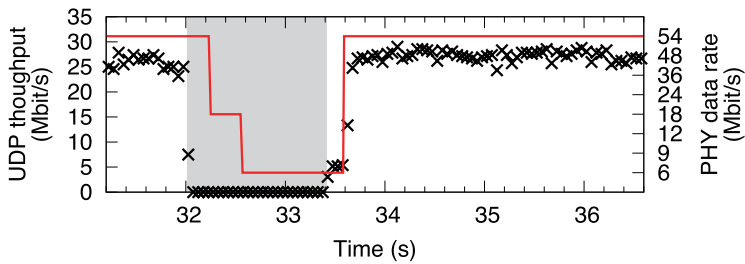

UDP throughput for every $0.050 \mathrm{~s}$ PHY data rate

(c) PID-based scheme without rectenna output for comparison

Fig. 5 UDP throughput and PHY data rate for each of the three PIDbased RASs, where the grey area indicates the time during which the DT is exposed to microwave radiation for MPT.

each of the Minstrel-based RASs. Except for the change in the link quality, the UDP throughput in Fig. 7(a) can be considered the same as that in Figs. 7(b) and 7(c). On the other hand, after the MPT is discontinued, a PHY data rate of less than $48 \mathrm{Mbit} / \mathrm{s}$ is sometimes used in Fig. 7(a), while that of $54 \mathrm{Mbit} / \mathrm{s}$ is always used in Figs. 7(b) and 7(c).

\section{Discussion}

Taking the observations in Sect. 4 into account, we discuss the advantages of the proposed scheme.

Exposure assessment based on the rectenna output allows a device equipped with the rectenna to precisely recognize in real-time that the device is exposed to microwave radiation for MPT. Here it should be noted that if a device is powered with MPT, the device has a rectenna installed. According to either Figs. 5(b) and 5(c) or Figs. 7(b) and 7(c), the UDP throughput of the proposed scheme can be regarded as the same as that of the RAS without rectenna output for comparison, except for the change in the quality of the link. However, the use of the scheme without rectenna output for comparison has the following two disadvantages. Firstly, as described in Sect. 1, because of the exposure assessment

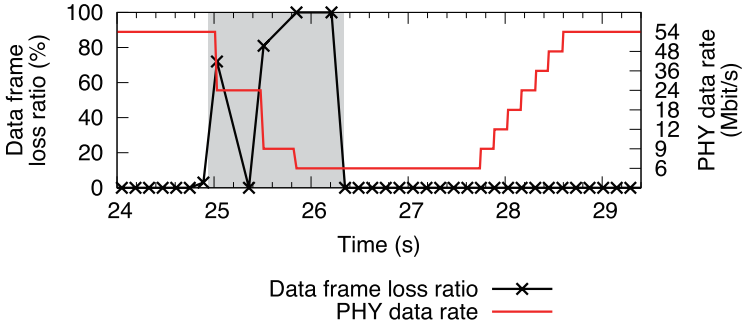

(a) PID

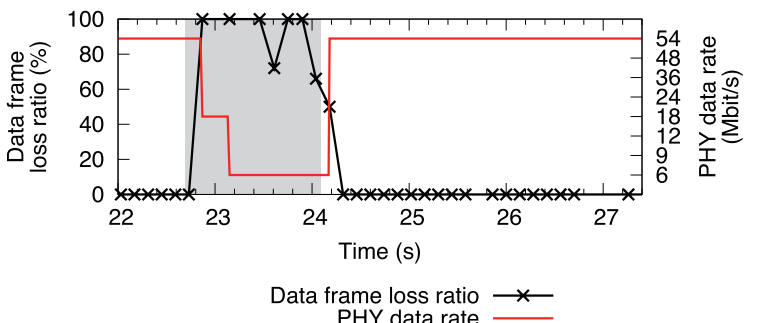

(b) PID-based proposed scheme

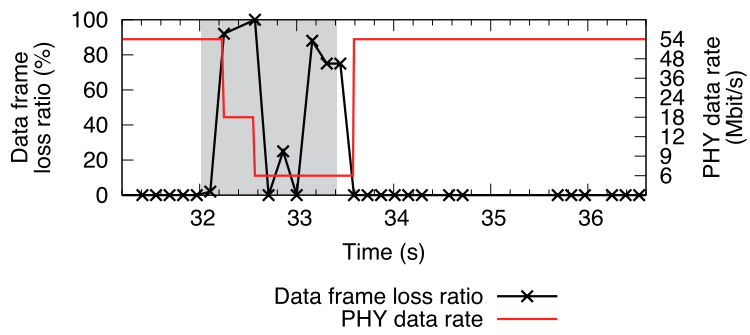

(c) PID-based scheme without rectenna output for comparison

Fig. 6 Data frame loss ratio and PHY data rate for each of the three PIDbased RASs, where the grey area indicates the time during which the DT is exposed to microwave radiation for MPT.

based on the data frame loss ratio, when severe SNR degradation occurs at the receiver, the station based on the scheme erroneously determines that it is exposed to microwave radiation for MPT even when it is not. Secondly, because of the exposure assessment based on the number of transmitted data frames, when the station transmits data frames under unsaturated traffic conditions or when there are other nodes that operate in the same band as the station, the station can erroneously determine that it is exposed to microwave radiation for MPT even when this is not the case. The cause of this is that the number of transmitted data frames fluctuates depending both on the traffic conditions and on the contention level.

Using historical data corresponding to the state of exposure to microwave radiation for MPT has the following advantages in comparison with using continuously historical data stored in a single memory.

- After discontinuation of the MPT, the high PHY data rate is maintained, and thus throughput degradation does not occur. Compared with Fig.5(b), Fig. 5(a) shows that the PHY data rate was reduced over the period from 24.8 to $26.2 \mathrm{~s}$ temporarily, after which a PHY data rate below $48 \mathrm{Mbit} / \mathrm{s}$ was used over the pe- 


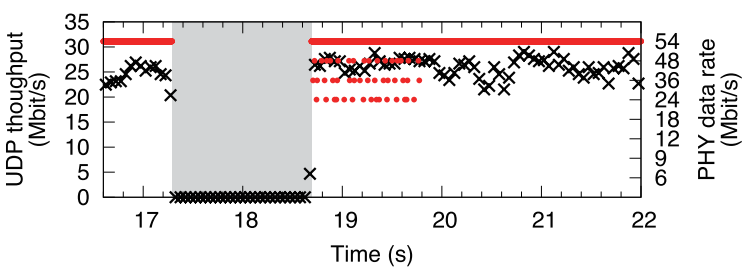

UDP throughput for every $0.050 \mathrm{~s} \quad \mathrm{P}$

(a) Minstrel

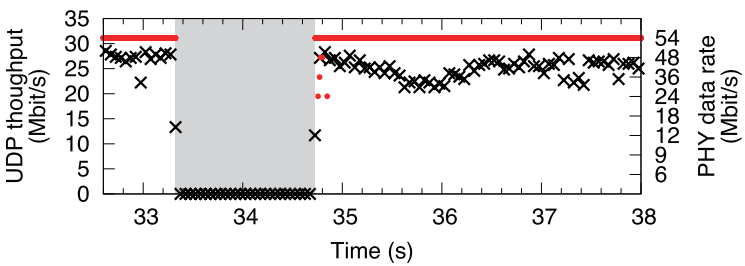

UDP throughput for every $0.050 \mathrm{~s} \quad \mathrm{X}$

(b) Minstrel-based proposed scheme

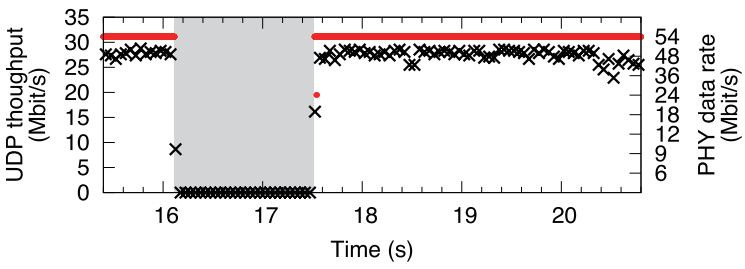

UDP throughput for every $0.050 \mathrm{~s} \quad \mathrm{x}$ PHY data rate

(c) Minstrel-based scheme without rectenna output for comparison

Fig. 7 UDP throughput and PHY data rate for each of the three Minstrelbased RASs, where the grey area indicates the time during which the DT is exposed to microwave radiation for MPT.

riod from 26.2 to 28.6 s. On the other hand, Fig. 5(b) shows that a PHY data rate below $48 \mathrm{Mbit} / \mathrm{s}$ was not used over the period from 24.2 to $26.6 \mathrm{~s}$.

- Using historical data corresponding to the exposure assessment result prevents the content of historical data from being corrupted. Compared with Fig. 7(b), Fig. 7(a) shows that a PHY data rate below $48 \mathrm{Mbit} / \mathrm{s}$ was sometimes used over the period from 18.7 to 19.8 s. On the other hand, Fig. 7(b) shows that a PHY data rate below $48 \mathrm{Mbit} / \mathrm{s}$ was not used over the period from 34.9 to $35.8 \mathrm{~s}$. The reason for this is that in Fig. 7(a), the contents stored in $M_{\mathrm{NE}}$ were corrupted due to a data frame loss when the DT is exposed to microwave radiation for MPT.

\section{Conclusion}

This paper proposed an RAS based on exposure assessment using rectenna output for a WLAN station powered with MPT. The objective of the proposed scheme was to prevent the throughput degradation that occurs when the station is exposed to microwave radiation for MPT. This objective was achieved by utilizing the rectenna output to assess



(a) Minstrel



Data frame loss ratio $\longrightarrow$

(b) Minstrel-based proposed scheme

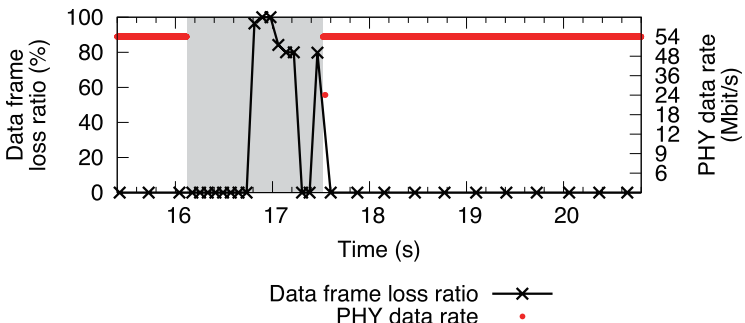

(c) Minstrel-based scheme without rectenna output for comparison

Fig. 8 Data frame loss ratio and PHY data rate for each of the three Minstrel-based RASs, where the grey area indicates the time during which the DT is exposed to microwave radiation for MPT.

whether the station is exposed to microwave radiation for MPT. Note that a device powered with MPT has a rectenna. The proposed scheme is based on the use of two independent memories in which historical data for rate adaptation were stored with the aim of mitigating the PHY data rate degradation when the station is exposed to microwave radiation for MPT. The historical data stored in the two memories were used to determine when the station was either exposed or not exposed to microwave radiation for MPT. We implemented the proposed scheme on a station using a commercial WLAN device and a widely used device driver. The results confirmed the effectiveness of the proposed scheme.

\section{Acknowledgment}

This work was supported in part by JSPS KAKENHI Grant Number 24360149. This experiment was carried out using the Microwave Energy Transmission Laboratory (METLAB) system of the Research Institute for Sustainable Humanosphere at Kyoto University.

\section{References}

[1] N. Shinohara, "Power without wires," IEEE Microw. Mag., vol.12, 
no.7, pp.S64-S73, Dec. 2011.

[2] N. Imoto, S. Yamashita, T. Ichihara, K. Yamamoto, T. Nishio, M. Morikura, and N. Shinohara, "Experimental investigation of co-channel and adjacent channel operations of microwave power and IEEE $802.11 \mathrm{~g}$ data transmissions," IEICE Trans. Commun., vol.E97-B, no.9, pp.1835-1842, Sept. 2014.

[3] A. Kamerman and L. Monteban, "WaveLAN-II: A high-performance wireless LAN for the unlicensed band," Bell Labs Tech. J., vol.2, no.3, pp.118-133, June 1997.

[4] G. Holland, N. Vaidya, and P. Bahl, "A rate-adaptive MAC protocol for multi-Hop wireless networks," Proc. 7th Annual International Conference on Mobile Computing and Networking-MobiCom'01, pp.236-251, 2001.

[5] "Madwifi Onoe specification," http://sourceforge.net/projects/ madwifi/

[6] J.C. Bicket, "Bit-rate selection in wireless networks," MIT Master's Thesis, Feb. 2005.

[7] Q. Pang, V.C.M. Leung, and S.C. Liew, "A rate adaptation algorithm for IEEE 802.11 WLANs based on MAC-layer loss differentiation," Proc. 2nd International Conference on Broadband Networks, 2005, vol.1, pp.659-667, 2005.

[8] J. Kim, S. Kim, S. Choi, and D. Qiao, "CARA: Collision-aware rate adaptation for IEEE 802.11 WLANs," Proc. IEEE INFOCOM 2006. 25TH IEEE International Conference on Computer Communications, pp.1-11, 2006

[9] S. Biaz and S. Wu, "Loss differentiated rate adaptation in wireless networks," Proc. 2008 IEEE Wireless Communications and Networking Conference, pp.1639-1644, 2008.

[10] S. Wu, S. Biaz, and H. Wang, "Rate adaptation with loss diagnosis on IEEE 802.11 networks," Int. J. Commun. Syst., vol.25, no.4, pp.515-528, April 2012.

[11] J. Zhang, K. Tan, J. Zhao, H. Wu, and Y. Zhang, "A practical SNR-guided rate adaptation," Proc. IEEE INFOCOM 2008 - The 27th Conference on Computer Communications, pp.2083-2091, 2008 .

[12] K. Pelechrinis, I. Broustis, S.V. Krishnamurthy, and C. Gkantsidis, "A measurement-driven anti-jamming system for 802.11 Networks," IEEE/ACM Trans. Netw., vol.19, no.4, pp.1208-1222, Aug. 2011.

[13] D. Aguayo, J. Bicket, S. Biswas, G. Judd, and R. Morris, "Link-level measurements from an $802.11 \mathrm{~b}$ mesh network," ACM SIGCOMM Computer Communication Review, vol.34, no.4, pp.121-132, 2004.

[14] “mac80211," http://wireless.kernel.org/en/developers/Documentatio $\mathrm{n} / \mathrm{mac} 80211 /$

[15] “PID," http://wireless.kernel.org/en/developers/Documentation/mac 80211/RateControl/PID

[16] "Minstrel specification," http://madwifi-project.org/svn/madwifi/ trunk/ath_rate/minstrel/minstrel.txt/

[17] W. Yin, P. Hu, J. Indulska, and K. Bialkowski, "Performance of mac80211 rate control mechanisms," Proc. 14th ACM International Conference on Modeling, Analysis and Simulation of Wireless and Mobile Systems - MSWiM'11, pp.427-436, 2011.

[18] D. Xia, J. Hart, and Q. Fu, "Evaluation of the minstrel rate adaptation algorithm in IEEE 802.11g WLANs," Proc. 2013 IEEE International Conference on Communications (ICC), pp.2223-2228, 2013.

[19] "Raspberry Pi model B+," http://www.raspberrypi.org/products/ model-b-plus/

[20] “ArduinoBoardUno," http://arduino.cc/en/Main/arduinoBoardUno/

[21] “Iperf," http://www.iperf.fr/

[22] "Wireshark," https://www.wireshark.org/



Shota Yamashita received the B.E. degree in electrical and electronic engineering from Kyoto University in 2013. He is currently studying toward the M.E. degree at the Graduate School of Informatics, Kyoto University. His research interests include microwave power transmission to WLAN devices. He is a student member of the IEEE.

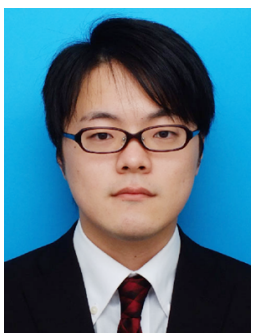

Koichi Sakaguchi received the B.E. degree in electrical and electronic engineering from Kyoto University in 2014. He is currently studying toward the M.E. degree at the Graduate School of Informatics, Kyoto University. His current research interests include fullduplex wireless communication systems.

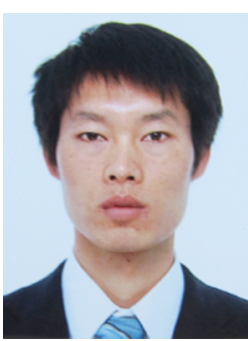

Yong Huang received the B.S. degree in integrated mechanical and electrical engineering from Jiangnan University, Jiangsu, China, in 2008, the M.S. degree in electric and electronic engineering from the University of Toyama, Toyama, Japan, in 2011, and is currently working toward the $\mathrm{Ph} . \mathrm{D}$. degree in electrical engineering at Kyoto University, Kyoto, Japan. His current research interests include rectifying circuit integration and power electronics of low-power rectenna design.

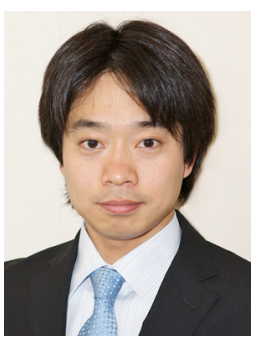

Koji Yamamoto received the B.E. degree in electrical and electronic engineering from Kyoto University in 2002, and the M.E. and Ph.D. degrees in informatics from Kyoto University in 2004 and 2005, respectively. From 2004 to 2005, he was a research fellow of the Japan Society for the Promotion of Science (JSPS). Since 2005, he has been with the Graduate School of Informatics, Kyoto University, where he is currently an associate professor. From 2008 to 2009 , he was a visiting researcher at Wireless@KTH, Royal Institute of Technology (KTH) in Sweden. His research interests include the application of game theory, spectrum sharing, and M2M networks. He received the PIMRC 2004 Best Student Paper Award in 2004, the Ericsson Young Scientist Award in 2006, and the Young Researcher's Award from the IEICE of Japan in 2008. He is a member of the IEEE. 


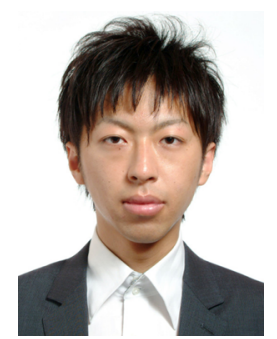

Takayuki Nishio received the B.E. degree in Electrical and Electronic Engineering from Kyoto University in 2010. He received the M.I. and Ph.D. degrees in Communications and Computer Engineering, Graduate School of Informatics from Kyoto University, Kyoto, Japan, in 2012 and 2013, respectively. He is currently an Assistant Professor in Communications and Computer Engineering, Graduate School of Informatics, Kyoto University. His current research interests include communication system design, protocol design particularly MAC and TCP, and QoS and resource management in wireless networks. He is a member of the IEEE.

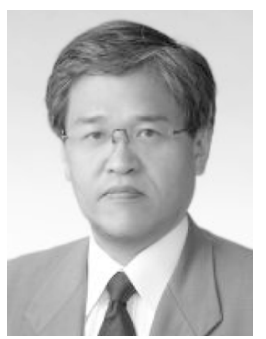

Masahiro Morikura received his B.E., M.E., and Ph.D. degrees in electronics engineering from Kyoto University, Kyoto, Japan in 1979, 1981 and 1991, respectively. He joined NTT in 1981, where he was engaged in the research and development of TDMA equipment for satellite communications. From 1988 to 1989, he was with the Communications Research Centre, Canada, as a guest scientist. From 1997 to 2002, he was active in the standardization of the IEEE 802.11a based wireless LAN. His current research interests include WLANs and M2M wireless systems. He received the Paper Award and the Achievement Award from IEICE in 2000 and 2006, respectively. He also received the Education, Culture, Sports, Science and Technology Minister Award in 2007 and Maejima Award in 2008. Dr. Morikura is now a professor in the Graduate School of Informatics, Kyoto University. He is a member of the IEEE.



Naoki Shinohara received the B.E. degree in electronic engineering, the M.E. and Ph.D. (Eng.) degrees in electrical engineering from Kyoto University, Japan, in 1991, 1993 and 1996, respectively. He was a research associate in the Radio Atmospheric Science Center, Kyoto University from 1998 . He was a research associate of the Radio Science Center for Space and Atmosphere, Kyoto University by recognizing the Radio Atmospheric Science Center from 2000, and there he was an associate professor since 2001. He was an associate professor in Research Institute for Sustainable Humanosphere, Kyoto University by recognizing the Radio Science Center for Space and Atmosphere since 2004. From 2010, he has been a professor in Research Institute for Sustainable Humanosphere, Kyoto University. He has been engaged in research on Solar Power Station/Satellite and Microwave Power Transmission system. He is IEEE MTT-S Technical Committee 26 (Wireless Power Transfer and Conversion) member, IEEE MTT-S Kansai Chapter TPC member, advisory committee member of IEEE Wireless Power Transfer Conference, executive editor of international journal of Wireless Power Transfer (Cambridge Press), Japanese committee C member of Radio Science for URSI, chair of technical committee on Wireless Power Transfer, communications society, IEICE, chair of Wireless Power Transfer Consortium for Practical Applications (WiPoT), and chair of Wireless Power Management Consortium (WPMc). 